\title{
Glucose oscillations, more than constant high glucose, induce p53 activation and a metabolic memory in human endothelial cells
}

\author{
B. Schisano - G. Tripathi - K. McGee • P. G. McTernan • \\ A. Ceriello
}

Received: 24 August 2010 / Accepted: 7 December 2010 /Published online: 2 February 2011

(C) Springer-Verlag 2011

\begin{abstract}
Aims/hypothesis Damage persists in HUVECs exposed to a constant high glucose concentration long after glucose normalisation, a phenomenon termed 'metabolic memory'. Evaluation of the effects of exposure of HUVECs to oscillating high glucose on the induction of markers of oxidative stress and DNA damage (phospho- $\gamma$-histone $\mathrm{H} 2 \mathrm{AX}$ and $\mathrm{PKC} \delta$ ) and onset of metabolic memory, and the possible role of the tumour suppressor transcriptional factor p53 is of pivotal interest.

Methods HUVECs were incubated for 3 weeks in 5 or $25 \mathrm{mmol} / \mathrm{l}$ glucose or oscillating glucose $(24 \mathrm{~h}$ in $5 \mathrm{mmol} / 1$ glucose followed by $24 \mathrm{~h}$ in $25 \mathrm{mmol} / \mathrm{l}$ glucose) or for 1 week in constant $5 \mathrm{mmol} / \mathrm{l}$ glucose after being exposed for 2 weeks to continuous $25 \mathrm{mmol} / \mathrm{l}$ high glucose or oscillating glucose. Transcriptional activity of $\mathrm{p} 53$ was also evaluated in the first $24 \mathrm{~h}$ after high glucose exposure.

Results High constant glucose upregulated phospho- $\gamma$ histone $\mathrm{H} 2 \mathrm{AX}$ and protein kinase $\mathrm{C}(\mathrm{PKC}) \delta$ compared with control. Oscillating glucose was even more effective than both normal and constant high glucose. Both constant and oscillating glucose resulted in a memory effect, which was more pronounced in the oscillating condition. Tran-
\end{abstract}

B. Schisano and G. Tripathi contributed equally to this study.

B. Schisano $\cdot$ G. Tripathi $\cdot$ K. McGee $\cdot$ P. G. McTernan

Clinical Science Research Institute (CSRI),

Warwick Medical School, University of Warwick,

University Hospital-Walsgrave Campus,

Coventry CV2 2DX, UK

\section{A. Ceriello $(\bowtie)$}

Insititut d'Investigacions Biomèdiques,

August Pi i Sunyer (IDIBAPS), Calle Mallorca 183, Piso P01, 08036 Barcelona, Spain

e-mail: aceriell@clinic.ub.es scriptional activity of p53 peaked $6 \mathrm{~h}$ after glucose exposure, showing a predicted oscillatory behaviour.

Conclusions/interpretation Exposure to oscillating glucose was more deleterious than constant high glucose and induced a metabolic memory after glucose normalisation. Hyperactivation of p53 during glucose oscillation might be due to the absence of consistent feedback inhibition during each glucose spike and might account for the worse outcome of this condition.

Keywords Cardiovascular disorders · Endothelial function · HUVECs · Hyperglycaemia - Metabolic memory · Oscillating glucose $\cdot$ p53 activation $\cdot$ Type 2 diabetes

$\begin{array}{ll}\text { Abbreviations } \\ \text { ATM } & \text { Ataxia-telangiectasia mutated } \\ \gamma \text {-H2AX } & \gamma \text {-Histone H2AX } \\ \text { HG } & \text { High glucose } \\ \text { HG-M } & \text { High glucose memory } \\ \text { NG } & \text { Normal glucose } \\ \text { OSG } & \text { Oscillating glucose } \\ \text { OsG-M } & \text { Oscillating glucose memory } \\ \text { PKC } & \text { Protein kinase C } \\ \text { PTEN } & \text { Phosphatase and tensin homologue } \\ \text { PUMA } & \text { p53 upregulated mediator of apoptosis } \\ \text { MDM2 } & \text { Murine double minute oncoprotein } \\ \text { ROS } & \text { Reactive oxygen species } \\ \text { TIGAR } & \text { TP53-induced glycolysis and apoptosis } \\ & \text { regulator }\end{array}$

\section{Introduction}

Since vascular tissue is unable to regulate passive inflow of glucose in a hyperglycaemic environment, the more glucose 
the endothelial cell is exposed to, the more is transported within the cell $[1,2]$. Glucose metabolism in hyperglycaemic conditions ultimately triggers the formation of a very high number of electron donors, such as NADH and $\mathrm{FADH}_{2}$ [3]. Inflow of such an elevated number of electrons to the mitochondrial respiratory chain leads to an increase in the voltage gradient across the mitochondrial membrane and its default. Electrons at this point are donated to molecules of oxygen, triggering the formation of a high number of superoxide anions [2, 3]. These highly reactive oxygen species (ROS) are then converted by the cellular antioxidant machinery into other oxidants, such as hydrogen peroxide $\left(\mathrm{H}_{2} \mathrm{O}_{2}\right)$ and peroxynitrite [4]. These molecules propagate oxidative damage to all cellular compartments, leading to lipid and protein oxidation [4].

Not surprisingly, one of the first consequences of excessive superoxide formation at the mitochondrial level is mitochondrial and genomic DNA damage $[5,6]$. One of the first cellular markers of DNA damage is phospho- $\gamma$ histone $\mathrm{H} 2 \mathrm{AX}(\gamma-\mathrm{H} 2 \mathrm{AX})$ [7]. Its formation is induced by activated protein kinase $\mathrm{C}(\mathrm{PKC})$ and ataxia-telangiectasia mutated (ATM) proteins [8, 9]. This histone becomes extensively phosphorylated within 1-3 min of DNA damage and evidence strongly supports its role in focus formation at sites of double-strand breaks [10]. Interestingly, changes in chromatin structure accompanying doublestrand break repair are also related to the presence of this phosphorylated histone [11].

ATM activation following genotoxic stress leads not only to formation of phospho- $\gamma-\mathrm{H} 2 \mathrm{AX}$, but also to p53 phosphorylation $[12,13]$. The role of the well known tumour suppressor transcription factor p53 in diabetes has long been undervalued. Research has recently focused on p53 activation in human endothelial cells exposed to high glucose levels [14], showing how p53-induced cellular senescence in vascular cells might contribute to accelerated ageing and atherosclerosis $[15,16]$. Furthermore, studies showed that inhibition of p53 activity in mouse adipose tissue markedly decreased the expression of proinflammatory cytokines and improved insulin resistance in mice with type 2 diabetes-like disease. Conversely, upregulation of p53 in adipose tissue caused an inflammatory response that led to insulin resistance [17].

Activation of p53 triggers upregulation of genes involved in apoptosis, such as PUMA (p53 upregulated mediator of apoptosis; also known as $B B C 3$ ) and PTEN (phosphatase and tensin homologue) [18, 19], and in senescence, such as p21 (also known as CDKN1A) [20], and of its feedback inhibitor, murine double minute oncoprotein (MDM2) [21]. Recently, it has also been shown that p53 regulates glucose metabolism via Tp53-induced glycolysis and apoptosis regulator (TIGAR) [22] and insulin sensitivity via PTEN, the main negative regulator of phosphorylation of the serine/threonine protein kinase AKT [17]. Moreover, studies suggest that p53 might perform its senescence and pro-apoptotic functions by directly signalling the mitochondria and inducing cytochrome $c$ release $[23,24]$. These data were confirmed by experiments showing p53 mobilisation to the mitochondrial membrane during oxidative stress induced by hyperglycaemia, leading to changes in mitochondrial membrane potential [25].

Interestingly, it has been shown that normalising the external glucose level does not switch off this intracellular pro-oxidant environment. This effect has been defined as 'metabolic memory' [26]. Studies on the metabolic memory began some decades ago, when persistence in the high production of basement membrane components (fibronectin, collagen IV), long after high glucose levels were normalised was shown in endothelial cells [27]. More recently, Ihnat et al. [28] have shown in cultured endothelial cells and in diabetic animals a ROS-mediated cellular persistence of vascular stress after glucose normalisation, involving persistent upregulation of markers of oxidative stress and pathways consistently involved in the pathogenesis of diabetic complications.

All these studies involved prolonged exposure of endothelial cells to constant high glucose levels. However, oscillations in glucose levels, like those daily experienced by diabetic patients, have been shown to have the most deleterious effects on the vascular endothelium in various studies $[29,30]$. For this reason, assessing p53 activation after prolonged exposure to constant and oscillating high glucose might give us insights into the mechanisms by which oscillations in glucose levels represent a more detrimental condition compared with constant high glucose exposure. Finally, this study also aims to evaluate whether prolonged exposure to glucose oscillations might generate, as well as constant high glucose, a metabolic memory when glucose levels are normalised.

\section{Methods}

Subjects and ethics HUVECs were extracted from ten umbilical cords obtained from patients of the Warwick University Hospitals with written informed consent, in accordance with local research ethics committee guidelines and with local ethics committee approval.

Cell culture and glucose treatment HUVECs were grown in MCDB-131 medium (Gibco Life Technologies, Grand Island, NY, USA) with $15 \%$ (wt/vol.) FBS (Biosera, Ringmer, UK), epidermal growth factor $(10 \mathrm{ng} / \mathrm{ml}$; Invitrogen, Paisley, UK), fibroblast growth factor $(5 \mathrm{ng} / \mathrm{ml}$; Invitrogen) and penicillin/streptomycin (Invitrogen). Cells 
were incubated in five different conditions: (1) 3 weeks in $5 \mathrm{mmol} / \mathrm{l}$ glucose (normal glucose, NG), (2) 3 weeks in $25 \mathrm{mmol} / 1$ glucose (high glucose, HG), (3) 3 weeks in oscillating glucose $(24 \mathrm{~h}$ in $25 \mathrm{mmol} / \mathrm{l}$ glucose media followed by $24 \mathrm{~h}$ in $5 \mathrm{mmol} / 1$ glucose; oscillating glucose condition, OsG), or (4) 1 week in normal glucose $(5 \mathrm{mmol} / \mathrm{l})$ after 2 weeks in oscillating high glucose $(24 \mathrm{~h}$ in $25 \mathrm{mmol} / 1$ followed by $24 \mathrm{~h}$ in $5 \mathrm{mmol} / 1$ glucose; oscillating glucose memory condition, OsG-M) and (5) 1 week in normal glucose $(5 \mathrm{mmol} / \mathrm{l})$ after being exposed for 2 weeks to continuous high glucose $(25 \mathrm{mmol} / \mathrm{l}$; high glucose memory condition, HG-M). In the controls, $20 \mathrm{mmol} / 1$ of mannitol (Sigma, St Louis, MO, USA) was added to normalise osmolarity. Fresh medium was added to the cells every day and $12 \mathrm{~h}$ before the end of the experiments. Protein from the OsG conditions was collected while the cells were in HG medium. This design is described in Fig. 1.

Western blotting Whole-cell lysates were made using MPER lysis buffer (Pierce, Rockford, IL, USA) and containing protease inhibitor cocktail (EMD Bioscience, Gibbstown, NJ, USA) and phosphatase inhibitors (Sigma). Equal amounts of protein lysates ( 20 or $30 \mu \mathrm{g}$ ); determined using the Micro BCA Protein Assay Kit (Pierce) were separated using $8-12 \%$ (wt/vol.) SDS-PAGE gels, transferred to $0.2 \mu \mathrm{mol} / 1$ nitrocellulose (Schleicher and Schuell, Keene, $\mathrm{NH}, \mathrm{USA}$ ), blocked in SuperBlock (Pierce), incubated with primary antibodies, either overnight at $4^{\circ} \mathrm{C}$ or for $2 \mathrm{~h}$ at

Experimental design

$1 / 2$. Continuous normal glucose (NG) or high glucose (HG)

21 days

Normal glucose or high glucose

3. Oscillating glucose ( $24 \mathrm{~h} \mathrm{HG} / 24 \mathrm{~h} \mathrm{NG})$ : OsG

21 days

Oscillating glucose

4. Oscillating glucose (24 h HG/24 h NG) + NG: OsG-M

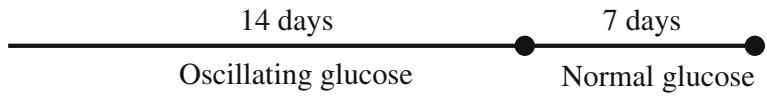

5. Continuous high glucose + NG: HG-M

$\stackrel{14 \text { days }}{\text { High glucose }} \underset{\text { Normal glucose }}{ }$

Fig. 1 Schematic representation of the five different conditions examined in this study. Cells were exposed to (1) 3 weeks in $5 \mathrm{mmol} / 1$ glucose $(\mathrm{NG})$; (2) 3 weeks in $25 \mathrm{mmol} / 1$ glucose (HG); (3) 3 weeks in oscillating glucose, i.e. alternating $24 \mathrm{~h}$ periods in $\mathrm{HG}$ and NG (OsG); (4) 2 weeks of OsG followed by 1 week of NG (OsG-M); (5) 2 weeks in HG followed by 1 week in NG (HG-M) room temperature, washed three times in Tris-buffered saline to which $0.25 \%$ Tween-20 secondary antibodies (Cell Signaling Technology, Danvers, MA, USA) had been added, washed again with $0.25 \%$ Tween- 20 , to which SuperSignal Dura chemiluminescence substrate (Pierce Chemical) had been added and subjected to digital imaging using a charge-coupled device camera. In all cases, equal loading was confirmed by probing blots against $\beta$-actin. Primary antibodies and dilutions used were as follows: phospho p53 ser15 (1:800; Cell Signaling Technology); PUMA (1:1,000; Cell Signaling Technology); p21 (1:1,000; Cell Signaling Technology); TIGAR (1:500; Abcam, Cambridge, MA, USA); PKC 8 (1:1,000; Cell Signaling Technology); phospho- $\gamma$ H2AX (1:800; Cell Signaling Technology); and Actin (1:2,000; Cell Signaling Technology).

Extraction of RNA and quantitative RT-PCR RNA was extracted from whole cells (RNeasy Lipid Tissue Kit; Qiagen, Crawley, UK), which included a DNase (Sigma) digestion step to remove any contaminating genomic DNA. RNA (1 mg) from each sample was reverse transcribed according to the manufacturer's instructions. Real-time PCR was performed in a reaction buffer containing TaqMan Universal PCR Master Mix (Applied Biosystems, Carlsbad, CA, USA), 150 ng cDNA and a pre-optimised primer and probe Gene Expression Assay (Applied Biosystems) specific for the genes. All reactions were multiplexed with the housekeeping gene $18 \mathrm{~S}$, provided as a pre-optimised control probe (Applied Biosystems). Data were obtained as $\mathrm{C}_{\mathrm{t}}$ values.

ROS fluorescence assay On the day preceding the end of the experiment, HUVECs were plated at a density of 7,000 cells $(70 \%$ confluence) on to white 96-well microplates with clear bottoms in $5 \mathrm{mmol} / \mathrm{l}$ medium. At the end of the experiment, medium was removed from the cells and $2 \mu \mathrm{g} / \mathrm{ml}$ of the cell-permeable $\mathrm{CM}-\mathrm{H}_{2}$ DCFDA probe (Molecular Probes-Invitrogen, Eugene, OR, USA) in Hanks' balanced salt solution was added to each well of the plates and incubated for $15 \mathrm{~min}$ at $37^{\circ} \mathrm{C}$ as previously described [31]. Background levels of the $\mathrm{CM}-\mathrm{H}_{2}$ DCFDA probe were assessed and subtracted from each reading. Fluorescence intensity was then measured using a plate reader (BMG Labtech, Durham, NC, USA) using $488 \mathrm{~nm}$ excitation and $530 \mathrm{~nm}$ emission filters.

Statistical analysis SPSS version 14.0 (SPSS, Woking, UK) was used to analyse data. Results are expressed as mean \pm SD. Groups were compared using two-way ANOVA, and the Bonferroni-Dunn post hoc test was performed on raw data. The two-tailed Student's $t$ test was used to assess differences between control and treated samples. $p<0.05$ was considered statistically significant. At 
least three independent experiments were performed in triplicate to ensure reproducibility.

\section{Results}

Assessment of p53 activation in HUVECs exposed to continuous high or oscillating glucose and its persistence after glucose normalisation Initial Western blotting experiments examined whether HUVECs exposed to chronic oscillating glucose $(\mathrm{OsG})$ would mediate differential upregulation of the active form of p53 and downstream signalling factors, compared with endothelial cells exposed to NG and/or continuous HG. Additional studies examined whether this upregulation in p53 would persist after 1 week of glucose normalisation. We therefore measured p53 phosphorylation on serine 15, phosphorylation being generated by ATM as a consequence of DNA damage [13] (Fig. 2a). Changes in the following p53 downstream signalling factors were measured: PTEN (Fig. 2a), a mediator in apoptosis and the main cellular inhibitor of AKT activation [19]; PUMA (Fig. 2c), which is involved in apoptosis [18]; p21 (Fig. 2d), involved in the regulation of growth and cellular senescence [20]; and TIGAR (Fig. 2e), involved in apoptosis and the regulation of glucose metabolism [22].

In the HG condition significant phosphorylation of p53 on serine 15 was observed compared with cells treated with NG. Exposure of cells to OsG induced significant upregulation of the phosphorylated form of p53 in comparison with cells treated with HG $(p<0.01)$ or NG $(p<0.001)$. p53 activation remained significantly upregulated after 1 week of glucose normalisation in cells previously exposed to OsG-M $(p<0.05)$, but not in cells exposed to HG-M.

Consequently, all p53-induced proteins examined (PTEN, p21, PUMA and TIGAR) remained significantly upregulated in cells treated with HG (PTEN, $p<0.01$; PUMA, p21 and TIGAR, $p<0.001)$ and OsG $(p<0.001)$ compared with cells exposed to NG. For PUMA, p21 and TIGAR, significant protein expression upregulation was noted in cells exposed to OsG compared with cells treated with $\mathrm{HG}$ alone $(p<0.01)$. With the only exception of PTEN, for which significant upregulation compared with $\mathrm{NG}$ once glucose levels were normalised was present in OsG-M $(p<0.05)$ but not in HG-M, all the p53-induced proteins examined were still significantly upregulated compared with NG in both OsG-M $(p<0.01)$ and HG-M (PUMA $p<0.03$; 21 and TIGAR, $p<$ $0.01)$. In particular, PUMA upregulation was significantly higher in OsG-M compared with HG-M $(p<0.02)$.

Assessment of oxidative stress markers in HUVECs exposed to continuous high or oscillating glucose and their persistence after glucose normalisation Western blotting was used to assess other markers of oxidative stress independently of p53. These studies examined the phosphorylated form of $\gamma$-H2AX on serine 139 [7] (Fig. 3a), as a marker of DNA damage, and $\mathrm{PKC} \delta$, an isoform of $\mathrm{PKC}$, which has been shown to be upregulated following a hyperglycaemic event that also induces oxidative stress [32] (Fig. 3b).
Fig. 2 Evidence of higher upregulation of the p53 pathway during glucose oscillation and of persistent induction of stress markers once glucose has been normalised. a Phospho-p53; b PTEN; c PUMA; d p21; e TIGAR. HUVECs were cultured in the five conditions described in Fig. 1. Whole-cell lysates were made and Western blots run as described in Methods. Data are mean \pm SD of the densitometry values of the bands. $* p<0.05, * * p<0.01, * * * p<$ 0.001 vs control (NG). Asterisks over bars refer to differences between the conditions shown under the bar. $n=9$ a

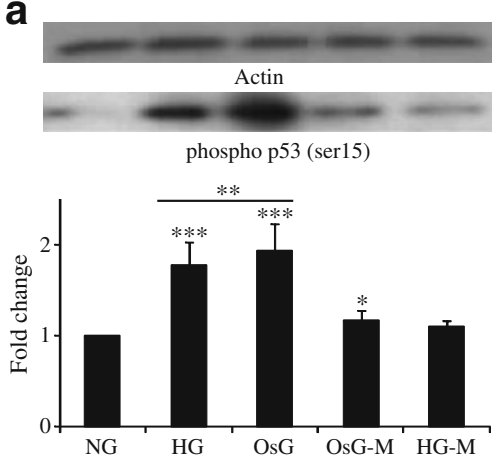

b

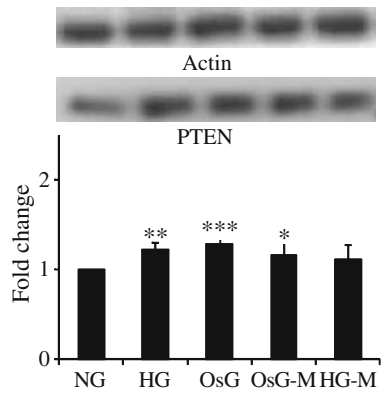

C
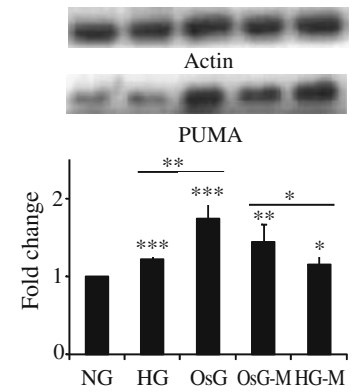

d

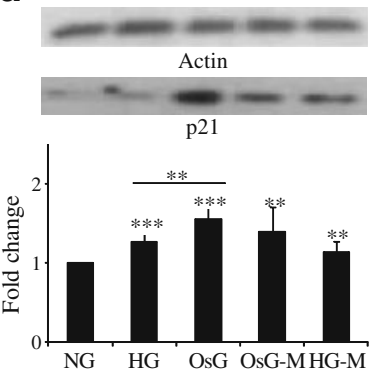

e

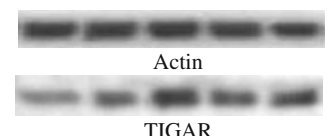

TIGAR

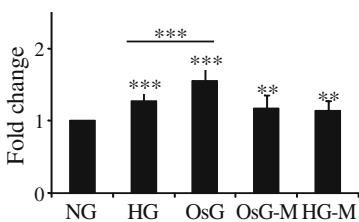


a
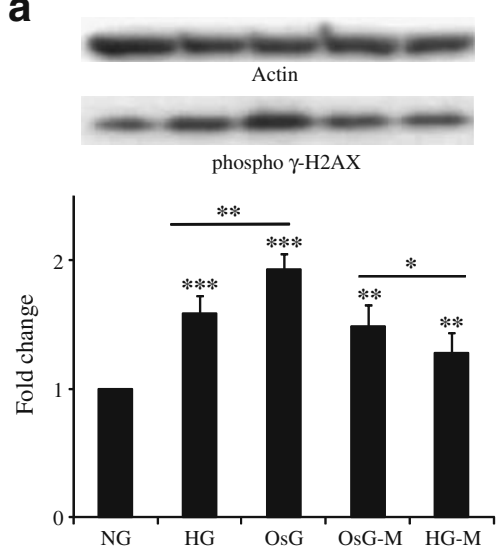

b

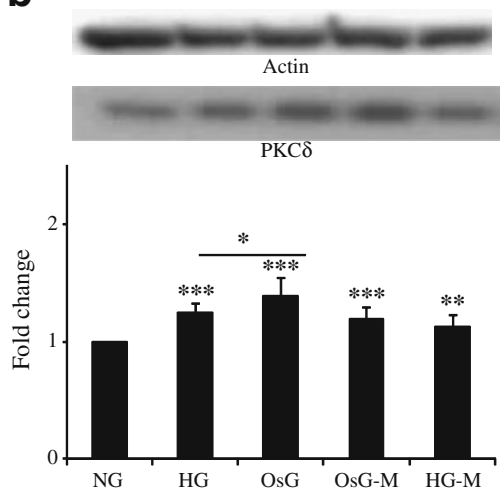

Fig. 3 Evidence of higher upregulation of markers of oxidative stress and DNA damage during glucose oscillations and of persistent induction of these stress markers once glucose has been normalised. a Phospho- $\gamma-\mathrm{H} 2 \mathrm{AX}$; b PKC $\delta$. HUVECs were cultured in the five conditions described in Fig. 1. Whole-cell lysates were made and Western blots run as described in the Methods. Data are mean $\pm \mathrm{SD}$ of the densitometry values of the bands. ${ }^{*} p<0.05, * * p<0.01, * * * p<$ 0.001 vs control (NG). Asterisks over bars refer to differences between the conditions shown under the bar. $n=9$

Both phospho- $\gamma-\mathrm{H} 2 \mathrm{AX}$ (Fig. 3a) and PKC $\delta$ (Fig. 3b) were significantly upregulated in cells treated with $\mathrm{HG}$ and OsG compared with cells treated with NG $(p<0.001)$. The two oxidative stress markers also showed significant upregulation in cells treated with OsG compared with cells treated with HG (phospho- $\gamma-\mathrm{H} 2 \mathrm{AX}, p<0.01$; PKC $\delta, \mathrm{p}<$ $0.05)$. Phospho- $\gamma-\mathrm{H} 2 \mathrm{AX}$ remained significantly upregulated after glucose normalisation in both OsG-M and HG-M $(p<0.01)$. Interestingly, phospho- $\gamma-\mathrm{H} 2 \mathrm{AX}$ also showed significant upregulation in cells treated with OsG-M compared with cells treated with HG-M $(p<0.05)$. PKC $\delta$ remained significantly upregulated in OsG-M $(p<0.001)$ and HG-M $(p<0.01)$ compared with cells treated with NG.

p53 transcriptional activity and MDM2 feedback inhibition after glucose exposure To assess whether p53-induced gene upregulation is limited in time by its feedback inhibition, we investigated the expression of MDM2 and other p53- induced genes ( 221, PUMA and TIGAR) in the first $24 \mathrm{~h}$ after constant HG exposure (Fig. 4). All genes induced by p53 that were analysed in this experiment showed significant upregulation at $3,6(p<0.01)$ and $9 \mathrm{~h}(p<0.05)$ compared with the control $(0 \mathrm{~h}$ time point), and at $6 \mathrm{~h}$ compared with $3,9,12$ and $24 \mathrm{~h}(p<0.05)$. Significant upregulation compared with the control was still present at $12 \mathrm{~h}$ for MDM2 and at $24 \mathrm{~h}$ for PUMA $(p<0.05)$.

Intracellular ROS production in continuous high and chronic oscillating glucose and after glucose normalisation Cells grown for 21 days in HG and OsG showed significant upregulation of ROS production compared with the control (Fig. $5 ; p<0.001$ ). Significant upregulation of ROS production was also observed in OsG compared with HG $(p<0.05)$.

ROS remained significantly upregulated compared with the control following glucose normalisation in HG-M ( $p<$ $0.01)$. However, ROS production remained increased after glucose normalisation even when cells were previously exposed to oscillating glucose (OsG-M, $p<0.001)$, showing significant upregulation compared with HG-M $(p<0.05)$.

\section{Discussion}

This study was able to confirm that a persistence, or cellular memory, of high glucose stress exists after glucose levels are normalised in endothelial cells, for oxidative stress (ROS production, phosphorylated form of histone $\gamma$-H2AX on serine 139, and $\mathrm{PKC} \delta$ ). However, this study shows for the first time the persistence of oxidative stress even when endothelial cells are exposed to a period of oscillating glucose. Moreover, it also demonstrated that chronic high glucose and oscillating glucose activate the tumour suppressor p53 and several proteins that are regulated by $\mathrm{p} 53$. This upregulation, except for PTEN, was more enhanced in oscillating glucose than in constant high glucose. The upregulation of p53 and of the related proteins persisted even when cells were grown in normal glucose after exposure to oscillating high glucose. Finally, it was evident that $6 \mathrm{~h}$ of high glucose was enough to induce the activation of p53 and of several related proteins.

Interestingly, this study confirmed that exposure to chronic oscillation in the glucose level represents a more detrimental condition for human endothelial cells compared with constant high glucose $[29,30]$. We observed that ROS production and subsequently markers of DNA damage and oxidative stress (other than those involved in the p53 pathway), such as phospho- $\gamma-\mathrm{H} 2 \mathrm{AX}$ and $\mathrm{PKC} \delta$, are significantly upregulated in oscillating glucose compared with constant high glucose. Moreover, in endothelial cells where glucose was normalised after prolonged exposure to 
Fig. 4 Real-time PCR showing the effect of high glucose on the transcription of genes induced by p53 in HUVECs. Cells were exposed to $25 \mathrm{mmol} / \mathrm{l}$ constant high glucose up to $24 \mathrm{~h}$ and RNA was extracted at $3,6,9,12$ and $24 \mathrm{~h}$ time points. RNA was extracted as described in the Methods. Data are represented as fold changes compared with control $(0 \mathrm{~h})$. Black columns, $M D M 2$; diagonally hatched columns, PUMA; vertically hatched columns, TIGAR; stippled columns, $p 21 .{ }^{*} p<0.05$ and $* * * p<0.001$ vs control (NG, 0 h); ${ }^{\dagger} p<0.05$ vs $3,9,12$ and 24 h. $n=9$

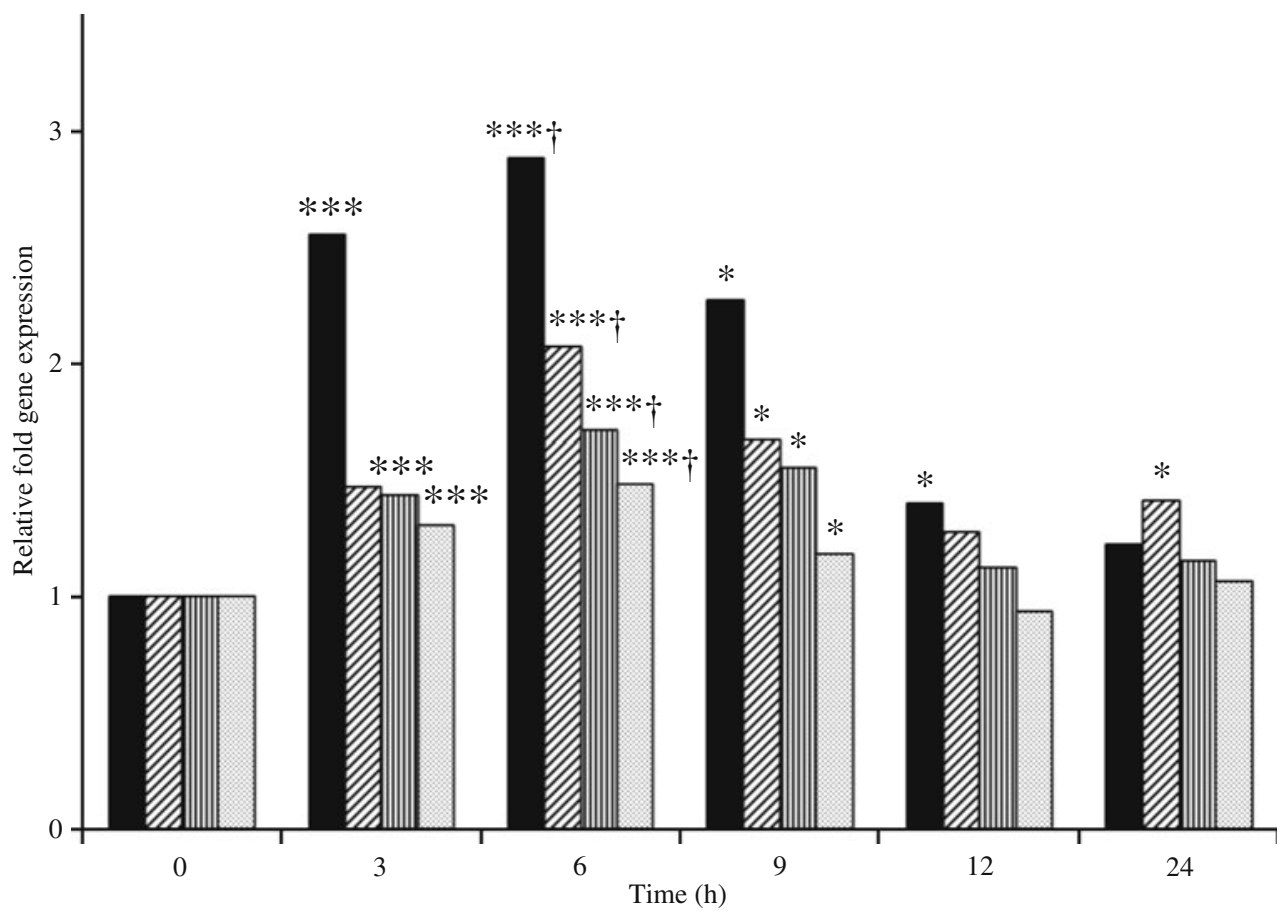

oscillating high glucose, we observed higher expression of these markers compared with cells that had been exposed to constant high glucose levels.

It has been shown recently that intermittent high glucose exacerbates the aberrant production of adiponectin and resistin through mitochondrial superoxide overproduction in adipocytes [33]. What generates this persistent dysfunction in the mitochondria after prolonged exposure to high glucose levels and especially after glucose oscillations is still not well understood.

Understanding the mechanism underlying the deleterious effect of glucose oscillations on vascular cells is of pivotal importance. While treatment in diabetic patients would help to minimise daily marked oscillations in plasma glucose values, in non-diabetic people glucose plasma concentrations are always within a narrow range [34]. This has raised the hypothesis that glucose variability, particularly after meal ingestion, may favour the development of complications in diabetes [35, 36]. Furthermore, our data suggest that glucose variability may also induce an even more deleterious metabolic memory compared with constant high glucose exposure. Persistence of epigenetic changes after a period of days during which cells were exposed in vitro to high glucose for only $16 \mathrm{~h}$ was first suggested by El-Osta et al. [37], and by our group in vivo; in normal participants we observed a carry-over effect of oscillating glucose in terms of endothelial dysfunction and nitrotyrosine generation [38]. While El-Osta et al. analysed the impact of the memory effect after a spike of high glucose lasting a few hours, we have tried to reproduce what might happen in poorly controlled diabetic patients, in whom consecutive long-lasting oscillations in glucose values are observed.

Interestingly, our data show hyperactivation of p53 during glucose oscillations and the study of $\mathrm{p} 53$ regulation is therefore of particular interest during oscillating glucose conditions. This transcription factor, as shown previously, is characterised by oscillatory activity [39-41], due to p53induced upregulation and its feedback inhibitor MDM2. As the data in Fig. 4 show, cells exposed to prolonged constant high glucose adapt to this condition following activation of the feedback inhibitor MDM2. On the other hand, glucose oscillations, where MDM2 feedback inhibitory activity cannot be sustained over time, are associated with significantly upregulated p53 activity and increased protein levels

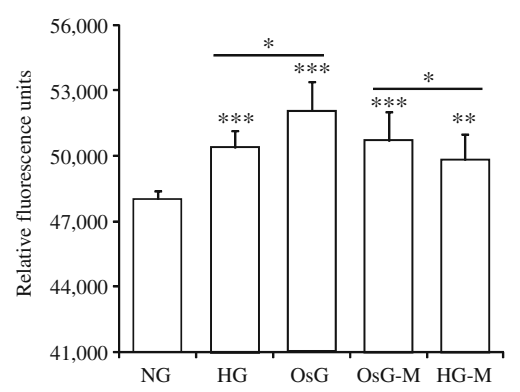

Fig. 5 Evidence of higher ROS build-up during glucose oscillations and its persistence once glucose levels have been normalised. Cells were grown in the conditions shown in Fig. 1. Cells were loaded with $2 \mu \mathrm{g} / \mathrm{ml}$ of CM- $\mathrm{H}_{2} \mathrm{DCFDA}$ for $15 \mathrm{~min}$ at $37^{\circ} \mathrm{C}$ and fluorescence was measured as described in Methods. Data are mean $\pm \mathrm{SD}$ of relative fluorescence units. ${ }^{*} p<0.05,{ }^{* *} p<0.01,{ }^{* * *} p<0.001$ vs control (NG). Asterisks over bars refer to differences between the conditions shown under the bar. $n=9$ 
of p21, PUMA, PTEN and TIGAR. These proteins have been shown to be responsible for cell growth arrest and induction of apoptosis. In summary, in constant high glucose, after a certain time and a certain number of $\mathrm{p} 53 /$ MDM2 oscillations, a steady state is reached for these two proteins, whereas under oscillating glucose conditions each glucose spike results in strong p53 transcriptional activity in the absence of established MDM2 inhibition. Interestingly, upregulation of p53-induced proteins persisted even after glucose normalisation and was higher when glucose was normalised after exposure to oscillating glucose rather than after exposure to constant high glucose. This might occur because overactivation and subsequent increased localisation of $\mathrm{p} 53$ in mitochondria [23, 24] during glucose oscillations might induce worse mitochondrial function, leading to greater mitochondrial dysfunction, subsequent ROS production and DNA damage that persists even when glucose levels are normalised.

Mitochondrial DNA damage persists after glucose normalisation [42]. It is therefore possible that the persistent upregulation of oxidative stress and DNA damage markers, once the glucose level is normalised, might be caused by the action of p53 on the mitochondria and thereby be increased by glucose variability.

In conclusion, large randomised studies have established that early intensive glycaemia control reduces the risk of diabetic complications - both microvascular and macrovascular - in both type 1 and type 2 diabetes [43]. However, epidemiological and prospective data support a long-term influence of early metabolic control on clinical outcomes [43]. This phenomenon has recently been described as 'metabolic memory' [28]. Furthermore, evidence suggests that glucose variability may also be an independent risk factor for cardiovascular complications in diabetes [35, 36, 44-46]. In this study we show that prolonged exposure to oscillating glucose generates a more detrimental condition in terms of ROS production, oxidative stress and DNA damage, leading to a memory effect of higher intensity than exposure to constant high glucose. We also show that p53 is overactivated by glucose oscillation, probably due to suboptimal feedback inhibition during the low glucose phases, and that its activation persists after glucose normalisation. The effect of p53 activity on mitochondrial function, which could be the cause of persistent damage when glucose is normalised, should be further investigated.

Acknowledgements We would like to acknowledge Novo Nordisk for their grant support of this work, all members of the Diabetes Group and the staff at the CSRI, Warwick Medical School. We would also like to thank Research Councils UK (RCUK) for a fellowship that has funded G. Tripathi.

Duality of interest The authors declare that there is no duality of interest associated with this manuscript.

\section{References}

1. Kaiser N, Sasson S, Feener EP et al (1993) Differential regulation of glucose transport and transporters by glucose in vascular endothelial and smooth muscle cells. Diabetes 42:80-89

2. Brownlee M (2001) Biochemistry and molecular cell biology of diabetic complications. Nature 414:813-820

3. Korshunov SS, Skulachev VP, Starkov AA (1997) High protonic potential actuates a mechanism of production of reactive oxygen species in mitochondria. FEBS Lett 416:15-18

4. Boveris A, Valdez LB, Zaobornyj T, Bustamante J (2006) Mitochondrial metabolic states regulate nitric oxide and hydrogen peroxide diffusion to the cytosol. Biochim Biophys Acta 1757:535-452

5. Madsen-Bouterse SA, Zhong Q, Mohammad G, Ho YS, Kowluru RA (2010) Oxidative damage of mitochondrial DNA in diabetes and its protection by manganese superoxide dismutase. Free Radic Res 44:313-321

6. Nishikawa T, Edelstein D, Du XL et al (2000) Normalising mitochondrial superoxide production blocks three pathways of hyperglycaemic damage. Nature 404:787-790

7. Kuo LJ, Yang LX (2008) Gamma-H2AX - a novel biomarker for DNA double-strand breaks. In Vivo 22:305-309

8. An J, Huang YC, Xu QZ et al (2010) DNA-PKCs plays a dominant role in the regulation of $\mathrm{H} 2 \mathrm{AX}$ phosphorylation in response to DNA damage and cell cycle progression. BMC Mol Biol 11:18

9. Mah LJ, Vasireddy RS, Tang MM, Georgiadis GT, El-Osta A, Karagiannis TC (2010) Quantification of gammaH2AX foci in response to ionising radiation. J Vis Exp 38. doi:10.3791/1957

10. Paull TT, Rogakou EP, Yamazaki V, Kirchgessner CU, Gellert M, Bonner WM (2000) A critical role for histone H2AX in recruitment of repair factors to nuclear foci after DNA damage. Curr Biol 10:886-895

11. Kinner A, Wu W, Staudt C, Iliakis G (2008) Gamma-H2AX in recognition and signalling of DNA double-strand breaks in the context of chromatin. Nucleic Acids Res 36:5678-5694

12. Shrivastav M, Miller CA, De Haro LP et al (2009) DNA-PKcs and ATM co-regulate DNA double-strand break repair. DNA Repair 8:920-929

13. Kulkarni A, Das KC (2008) Differential roles of ATR and ATM in $\mathrm{p} 53$, Chk1, and histone $\mathrm{H} 2 \mathrm{AX}$ phosphorylation in response to hyperoxia: ATR-dependent ATM activation. Am J Physiol Lung Cell Mol Physiol 294:L998-L1006

14. Liu B, Chen Y, St Clair DK (2008) ROS and p53: a versatile partnership. Free Radic Biol Med 44:1529-1535

15. Ahima RS (2009) Connecting obesity, aging and diabetes. Nat Med 15:996-997

16. Yokoi T, Fukuo K, Yasuda O et al (2006) Apoptosis signalregulating kinase 1 mediates cellular senescence induced by high glucose in endothelial cells. Diabetes 55:1660-1665

17. Minamino T, Orimo M, Shimizu I et al (2009) A crucial role for adipose tissue $\mathrm{p} 53$ in the regulation of insulin resistance. Nat Med 15:1082-1087

18. Amaral JD, Xavier JM, Steer CJ, Rodrigues CM (2010) The role of p53 in apoptosis. Discov Med 9:145-152

19. Jung IL, Kang HJ, Kim KC, Kim IG (2010) PTEN/pAkt/p53 signalling pathway correlates with the radioresponse of non-small cell lung cancer. Int J Mol Med 25:517-523

20. Fragkos M, Jurvansuu J, Beard P (2009) H2AX is required for cell cycle arrest via the p53/p21 pathway. Mol Cell Biol 29:2828-2840

21. Wade M, Wang YV, Wahl GM (2010) The p53 orchestra: Mdm2 and Mdmx set the tone. Trends Cell Biol 20:299-309

22. Bensaad K, Tsuruta A, Selak MA et al (2006) TIGAR, a p53inducible regulator of glycolysis and apoptosis. Cell 126:107-120 
23. Zhao Y, Chaiswing L, Velez JM et al (2005) p53 translocation to mitochondria precedes its nuclear translocation and targets mitochondrial oxidative defense protein-manganese superoxide dismutase. Cancer Res 65:3745-3750

24. Chipuk JE, Green DR (2009) PUMA cooperates with direct activator proteins to promote mitochondrial outer membrane permeabilization and apoptosis. Cell Cycle 8:2692-2696

25. Ortega-Camarillo C, Guzmán-Grenfell AM, García-Macedo R et al (2006) Hyperglycemia induces apoptosis and p53 mobilization to mitochondria in RINm5F cells. Mol Cell Biochem 281:163-171

26. Ceriello A, Ihnat MA, Thorpe JE (2009) Clinical review 2: The 'metabolic memory': is more than just tight glucose control necessary to prevent diabetic complications? J Clin Endocrinol Metab 94:410-415

27. Roy S, Sala R, Cagliero E, Lorenzi M (1990) Overexpression of fibronectin induced by diabetes or high glucose: phenomenon with a memory. Proc Natl Acad Sci USA 87:404-408

28. Ihnat MA, Thorpe JE, Kamat CD et al (2007) Reactive oxygen species mediate a cellular 'memory' of high glucose stress signalling. Diabetologia 50:1523-1531

29. Quagliaro L, Piconi L, Assaloni R, Martinelli L, Motz E, Ceriello A (2003) Intermittent high glucose enhances apoptosis related to oxidative stress in human umbilical vein endothelial cells: the role of protein kinase $\mathrm{C}$ and $\mathrm{NAD}(\mathrm{P}) \mathrm{H}$-oxidase activation. Diabetes 52:2795-2804

30. Quagliaro L, Piconi L, Assaloni R et al (2005) Intermittent high glucose enhances ICAM-1, VCAM-1 and E-selectin expression in human umbilical vein endothelial cells in culture: the distinct role of protein kinase $\mathrm{C}$ and mitochondrial superoxide production. Atherosclerosis 183:259-267

31. Miao F, Gonzalo IG, Lanting L, Natarajan R (2004) In vivo chromatin remodeling events leading to inflammatory gene transcription under diabetic conditions. J Biol Chem 279:18091-1807

32. Geraldes P, Hiraoka-Yamamoto J, Matsumoto M et al (2009) Activation of PKC-delta and SHP-1 by hyperglycaemia causes vascular cell apoptosis and diabetic retinopathy. Nat Med 15:1298-1306

33. Sun J, Xu Y, Deng H, Sun S, Dai Z, Sun Y (2010) Intermittent high glucose exacerbates the aberrant production of adiponectin and resistin through mitochondrial superoxide overproduction in adipocytes. J Mol Endocrinol 44:179-185

34. Mazze RS, Strock E, Wesley D et al (2008) Characterizing glucose exposure for individuals with normal glucose tolerance using continuous glucose monitoring and ambulatory glucose profile analysis. Diabetes Technol Ther 10:149-159

35. Ceriello A, Ihnat M (2010) "Glycemic variability": a new therapeutic challenge in diabetes and the critical care setting. Diabet Med 27:862-867

36. Ceriello A (2005) Postprandial hyperglycaemia and diabetes complications: is it time to treat? Diabetes 54:1-7

37. El-Osta A, Brasacchio D, Yao D et al (2008) Transient high glucose causes persistent epigenetic changes and altered gene expression during subsequent normoglycemia. J Exp Med 205:2409-2417

38. Ceriello A, Esposito K, Piconi L et al (2008) Oscillating glucose is more deleterious on endothelial function and oxidative stress than mean glucose in normals and type 2 diabetic patients. Diabetes 57:1349-1354

39. Lahav G (2008) Oscillations by the p53-Mdm2 feedback loop. Adv Exp Med Biol 641:28-38

40. Ciliberto A, Novak B, Tyson JJ (2005) Steady states and oscillations in the p53/Mdm2 network. Cell Cycle 4:488-493

41. Lev Bar-Or R, Maya R, Segel LA, Alon U, Levine AJ, Oren M (2000) Generation of oscillations by the p53-Mdm2 feedback loop: a theoretical and experimental study. Proc Natl Acad Sci USA 97:11250-11255

42. Madsen-Bouterse SA, Mohammad G, Kanwar M, Kowluru RA (2010) Role of mitochondrial DNA damage in the development of diabetic retinopathy, and the metabolic memory phenomenon associated with its progression. Antioxid Redox Signal 13:797-805

43. Ceriello A (2010) Hyperglycaemia and the vessel wall: the pathophysiological aspects on the atherosclerotic burden in patients with diabetes. Eur J Cardiovasc Prev Rehabil 17(Suppl 1):S15-S19

44. Writing Team for the Diabetes Control and Complications Trial/ Epidemiology of Diabetes Interventions and Complications Research Group (2002) Effect of intensive therapy on the microvascular complications of type 1 diabetes mellitus. JAMA 287:2563-2569

45. Monnier L, Mas E, Ginet C et al (2006) Activation of oxidative stress by acute glucose fluctuations compared with sustained chronic hyperglycaemia in patients with type 2 diabetes. JAMA 295:1681-1687

46. Nalysnyk L, Hernandez-Medina M, Krishnarajah G (2010) Glycaemic variability and complications in patients with diabetes mellitus: evidence from a systematic review of the literature. Diabetes Obes Metab 12:288-298 